\title{
Investigación biomédica y política científica en España, 1961-1975
}

\section{María Jesús Santesmases}

Arbor CLXVIII, 662 (Febrero 2001), 273-286 pp.

El desarrollo de la biología molecular y, en general de las investigaciones biomédicas en España no puede comprenderse sin repasar el papel jugado por un conjunto de científicos y científicas españoles que trataban de desarrollar sus propios proyectos de investigación. Esos proyectos incluían lograr que la investigación a la que se dedicaban llegara formar parte de la agenda política, lo que les permitiría mejores dotaciones para sus investigaciones y un mayor protagonismo académico de las áreas experimentales a las que se dedicaban. Para ello emprendieron negociaciones con las autoridades de la política científica, y así actuaron como asesores y consejeros. La consecuencia de esas negociaciones, junto a las tendencias en materia de política científica en expansión por Europa tras la Segunda Guerra Mundial proporcionan un marco para comprender el origen de la actual comunidad científica española dedicaca a las investigaciones biomédicas.

El surgimiento de las disciplinas o áreas de especialización experimental suele ir acompañado de políticas, públicas o privadas, de patrocinio. En el siglo XX, y especialmente tras la Segunda Guerra Mundial, el Estado adquiere un protagonismo creciente en la promoción de la investigación científica y técnica a través de las subvenciones concedidas a centros, departamentos, grupos de investigación o a personas concretas ligadas a proyectos definidos previamente ${ }^{1}$. Esa guerra 
dejó un rastro imborrable en el desarrollo científico y técnico del siglo XX. Ese rastro consiste fundamentalmente en un discurso a favor de la ciencia básica, de aplicaciones inicialmente imprevisibles pero que los comités científicos especializados dedicados al esfuerzo científicotécnico de la guerra en Estados Unidos se encargaron de estimular con éxito. Fueron las investigaciones europeas las que EEUU aplicó durante la guerra, como en el caso de la física de altas energías y de la penicilina, por citar solo dos de los más famosos. Esa ciencia básica debía ser apoyada por inversiones públicas que facilitarían la modernización científica y técnica de los países, no sólo en lo que atañía a la formación de especialistas sino en lo que se refería, y quizá se consideraba más influyente, a la modernización industrial y al desarrollo económico que debía producirse en la posguerra de una Europa devastada.

Estado Unidos cumplió su parte por dos caminos: promovió su propio desarrollo científico y ejerció una influencia principal en la definición y puesta en marcha de políticas científicas en Europa a través de la OECE, primero, y de la OCDE después. Como oficina económica dedicada a distribuir los presupuestos del Plan Marshall, la OECE promovió la formación técnica y la modernización científica y tecnológica en los países europeos. $\mathrm{Y}$ aunque, como se sabe, España no recibió ninguna ayuda de ese plan que tanto contribuyó a la recuperación económica, industrial, tecnológica y científica de Europa, sí recibió esas influencias por varias vías, especialmente a partir de la firma del Acuerdo con los Estados Unidos firmado en $1953^{2}$.

En la definición de las acciones en materia de política científica en los países europeos, los científicos mismos tuvieron su parte, una de las principales. Puede decirse que su protagonismo fue comparable, si no mayor, a la letra de las normas aprobadas en los países las vías de promoción de la investigación: leyes y agencias, autoridades y consejos científicos actuaron en los procesos de negociación que condujeron a los usos hoy vigentes sobre las vías para financiar las investigaciones, centros y proyectos de investigación. Los científicos se convirtieron en agentes de la política científica y técnica con el mismo protagonismo con el que fueron beneficiarios de ella. Grupos compuestos por especialistas influyentes ejercerían como consejeros de los gobiernos para las políticas científicas nacionales y en materia de cooperación científica internacional desde la inmediata posguerra y más intensamente a partir de finales de los años 1950.

En este artículo trato de exponer el protagonismo científico y político-científico logrado por un conjunto de investigadores dedicados a 
la bioquímica y a la biología molecular en la puesta en marcha de una política gubernamental en España, la cual, aun con recursos escasos, llegaría a establecer los usos actuales en materia de financiación de la investigación. Su actividad como investigadores es inseparable de los conocimientos adquiridos a lo largo del periodo de formación postdoctoral en el extranjero, entre mediados de los 1950 y mediados de los 1960. Aprendieron más que técnicas y conocimiento biológico, volvieron con más bagaje que el bibliográfico y algún aparato nuevo inexistente en la España de entonces entre su equipaje. Lo más influyente que trajeron consigo fue un proyecto de investigación y la manera en la que había que lograr financiación para llevarlo a la práctica. Volvieron con un proyecto de promoción de un área susceptible de expansión en España, en sus estructuras académicas e investigadoras.

Para el logro de tal ambicioso objetivo contaron con una ayuda: el premio Nobel de Medicina concedido a Severo Ochoa en 1959. Aunque Ochoa ya era por entonces ciudadano de los Estados Unidos, su papel en la promoción de la investigación biomédica en España fue esencial $^{3}$, pero no fue la única influencia importante que esa área experimental recibió. Un conjunto de contextos propicios permitieron que su influencia fuera posible, y hasta eficaz. Ese contexto estaba constituido por un grupo reducido pero ambicioso de científicos decididos a hacer en España investigación original y al día y por una situación política que, aunque cambiante a lo largo del periodo que cubre este artículo, tuvo su continuidad. Pero los avatares políticos del largo periodo que fue el franquismo hicieron que un conjunto de planes puestos en circulación desde mediados de los años 60 no se plasmaran en centros concretos y ayudas económicas recibidas hasta al menos 1970, cuando no hasta 1975.

La política científica se construye en muy buena parte desde abajo, es decir, desde el trabajo diario de los laboratorios descritos por Latour ${ }^{4}$. La medidas que las autoridades políticas toman están directamente influidas por la comunidad científica, y por aquellas personas de entre sus miembros que desempeñan el papel, más o menos oficial según el periodo de que se trate, de consejeros o asesores. El hacer diario de la experimentación en la mesa de trabajo y todo lo que eso lleva consigo, que incluye diseño y dirección de las experimentaciones, formación y desarrollo de un equipo, búsqueda de financiación, estrategias de publicación, desarrollo de redes de influencia y contacto entre colegas dedicados a materias afines, intereses docentes, logros de reconocimiento académico: todo ello constituye la política científica. No es sólo su expresión, es el laboratorio quien inventa las estrategias, las pone en 
marcha, aprovecha mecanismos disponibles para ejercer influencia, para hacer prevalecer sus criterios y para actuar eficazmente frente a la autoridad política. En ese sentido los jefes de los equipos de investigación actúan como gestores científicos además de como experimentadores. En muchos casos, han dejado las experimentaciones a otros miembros de su grupo de trabajo, cuentan con una persona leal al frente del laboratorio con la que mantienen contacto permanente, mientras ellos, o ellas, desde la dirección, se encargan de hacer posibles esos experimentos, elaboran un discurso - referido al carácter más o menos aplicado de los temas de investigación de su equipo- en el que basan la búsqueda de financiación, escriben los trabajos para su publicación, corrigen los borradores de tesis doctorales, seleccionan a los becarios y mantienen reuniones con aquellos que pueden subvencionarles y con los que pueden intercambiar o compartir bienes, saberes, técnicas, aparatos, y hasta colaboradores.

Todo esto resulta una obviedad para la comunidad científica ${ }^{5}$. De evidencia comparable resulta el hecho de que los recursos económicos disponibles para la investigación definen de forma esencial las políticas. Cuando, durante la segunda mitad del franquismo, los recursos eran escasos, quizá la influencia de los líderes científicos era mayor que la actual: el grupo de especialistas que por sus intereses llegaran a influir en los criterios de adjudicación de recursos era menos numeroso que el que hoy pueda estar interesado en hacerlo, la comunidad científica era también menos numerosa y el sistema burocrático de toma de decisiones estaba menos dotado que en la actualidad. Pero es que, además, los avatares políticos situaron a un grupo de científicos dedicados a la experimentación biomédica muy cerca de la autoridad política, cuando no la detentaban ellos mismos. Y aun cuando no lograran un puesto político-científico influyente, el grupo de gentes expertas dedicadas a la bioquímica y a la biología molecular actuaron como asesores y desde esa función hacían la política científica, pues sus criterios para la asignación de esos recursos, por escasos que fueran, resultaban influyentes ${ }^{6}$.

Así, desde la dirección de un laboratorio que había que sacar adelante con cualquier medio al alcance, los líderes de los grupos que serían finalmente influyentes optaron, en primer lugar, por ayudas extranjeras. El programa de ayudas extramuros de los National Institutes of Health (EE.UU.) tuvo un papel principal, al conceder a un grupo reducido de experimentadores biomédicos subvenciones de cuantía incomparable a las ayudas que se concedían entonces en España. Entre los organismos españoles que mayor presupuesto dedicaron a las experimentaciones 
en bioquímica y biología molecular estaban el Fondo Nacional para la Investigación Científica y Técnica, la Junta de Energía Nuclear y la Fundación Juan March. Aquellas ayudas extranjeras proporcionaron al conjunto de especialistas que las lograron una legitimación científica, profesional, que procedía, como su formación y sus conocimientos científicos y de gestión de laboratorios, del extranjero. Esa legitimidad fue el arma principal para reclamar apoyos político-científicos en España: si habían sido reconocidos por comités científicos extranjeros de los más distinguidas agencias de financiación e investigación de los Estados Unidos ¿cómo podría no reconocérseles experiencia y autoridad experta en su propio país? A la búsqueda de ese reconocimiento se emprendieron algunas acciones que terminaron, en el caso de las ciencias biomédicas experimentales, por lograr una comunidad científica influyente, fruto, en muy buena parte, de aquellos esfuerzos tempranos de un conjunto de bioquímicos y biólogos moleculares que había obtenido ayudas de los Estados Unidos. Tal fue el caso de Alberto Sols, Manuel Losada, Juan Antonio Subirana y Jaume Palau, David Vázquez, Federico Mayor Zaragoza y Margarita Salas y Eladio Viñuela. Formados en el extranjero con algunos de los más prestigiosos expertos de su tiempo, volvían a España entre mediados de los años 1950 y mediados de los 1960 para hacer de sus grupos centros de referencia e influencia en la expansión de la experimentación en la que se habían especializado.

Mientras tanto, el ministerio de Manuel Lora-Tamayo (1962-1968) cambió de nombre al volver este de una reunión sobre política científica convocada por la OCDE en 1963 en París. De ministerio de Educación Nacional pasó a llamarse de Educación y Ciencia. El nombre era un símbolo de la intención del gobierno español de seguir, o al menos de hacer parecer que se seguían, algunas de las propuesta hechas en aquella primera reunión. Lora-Tamayo era catedrático de Química Orgánica de la Universidad de Madrid. Mientras fue ministro se creó en 1963 la Comisión Delegada del Gobierno de Política Científica y en 1964 el Fondo Nacional, ya mencionado. En 1963 el propio Lora pronunció una conferencia en la segunda reunión bioquímica española y apoyó la creación de la Sociedad Española de Bioquímica. Él mismo se había interesado por algunos enzimas, aunque finalmente uno de sus discípulos, Ángel Martín Municio, sería el primer catedrático de Bioquímica de la Facultad de Ciencias de Madrid y organizador de los primeros cursos que se impartieron en España sobre biología molecular.

Para el empuje a las experimentaciones biomédicas en España fue aún más influyente el nombramiento de José Luis Villar Palasí como 
ministro de Educación en 1968, tras la dimisión de Lora-Tamayo al no conseguir evitar la entrada de la policía en los edificios universitarios en plenas protestas estudiantiles. Para contrarrestar manifestaciones y protestas el nuevo ministro creó, recién nombrado, tres universidades y anunció una «reforma de la Universidad». Su equipo, formado por algunos expertos de UNESCO, parece haberle convencido de la necesidad de una reforma de todo el sistema educativo. Para la Universidad se señaló la conveniencia de solicitar asesoramiento de los científicos españoles instalados en el extranjero. Ochoa fue visitado y remitió al representante español que le inquiría sobre la posibilidad de que asesorara la reforma de los estudios de medicina, al bioquímico Alberto Sols. Pero también sugirió la conveniencia de contar con un centro de investigación dedicado a la biología molecular en España ${ }^{7}$.

Sols estaba relacionado, a través de su mujer Angelines Rodríguez-Candela, con algunos médicos influyentes -él mismo era licenciado en Medicina, aunque le gustaba, como a muchos bioquímicos desde que se inventó la disciplina, decir que carecía de conocimientos para tratar a enfermos-, como el endocrinólogo José Luis Rodríguez-Candela y el prestigioso clínico Gregorio Marañón, que le había señalado en la inauguración del Centro de Investigaciones Biológicas como una de las promesas científicas españolas. Tenía contacto permanente con Albareda, aunque desconfiaba de la ayuda que podía ofrecerle el secretario general del Consejo Superior de Investigaciones Científicas dado que Sols había abandonado el Opus Dei, organización religiosa de la que Albareda era un miembro conocido e influyente. El prestigio científico de Sols estaba basado en sus trabajos originales sobre el metabolismo de los hidratos de carbono, en un conjunto de investigaciones emprendidas por él y su grupo de trabajo desde sus inicios en la investigación fisiológica hasta sus aportaciones a la regulación metabólica ${ }^{8}$.

Las relaciones del NIH con España estaban al cargo del neurofisiólogo Antonio Fernández de Molina. Pero, según se recuerda, fue Carlos Asensio, a su vuelta de una estancia larga en Nueva York, quien dio el aviso sobre los programa extramuros de los $\mathrm{NIH}^{9}$. Optar a esas ayudas trienales y obtenerlas era no sólo una fuente de recursos económicos, era también un medio de dar a conocer los proyectos españoles en Estados Unidos, cuya élite científica seleccionaba, a través de los correspondientes comités, los proyectos a subvencionar. Optaban a estas ayudas aquellos de formación postdoctoral estadounidense. Y Sols fue el único que logró dos ayudas trienales consecutivas, a pesar del criterio general de que estas ayudas a científicos extranjeros se concedían una sóla vez. Ochoa, entre otros, apoyó explícitamente la solicitud de Sols ${ }^{10}$. 
Villar Palasí nombró rector de la Universidad de Granada a un joven catedrático de Bioquímica y Fisiología de la Facultad de Farmacia: Federico Mayor Zaragoza, quien comenzó así su carrera político-científica. Mayor ha contado que fueron los hermanos del ministro, dos bioquímicos, Vicente y Carlos, los que le aconsejaron el nombramiento. Carlos Villar se instaló en Estados Unidos, y Vicente Villar, en Barcelona, sería nombrado por su hermano presidente de la comisión gestora de la universidad Aiutónoma de Barcelona, una de las de reciente creación 11.

Alberto Sols y Carlos Asensio, que había sido su discípulo y colaborador, elaboraron el primer documento en el que se proponía un centro posgraduado de biología molecular. El hecho de que comenzaran algunas negociaciones fue posible a través de los contactos nacionales e internacionales que se establecieron para buscar apoyos al proyecto. Cuando Ochoa asumió publicamente el liderzago del proceso en sus declaraciones públicas a partir de 1969, estaba llamando la atención de las autoridades políticas, tanto como la de la ciudadanía.

Cuando el biólogo molecular e investigador del CSIC Eladio Viñuela, con quien colaboraba el técnico Javier Corral, asumió la dirección de facto para la puesta en marcha de un centro de biología molecular en Madrid estaba desempeñando un conjunto de funciones complejas que incluían tanto la coordinación del proyecto como su puesta en práctica en el trabajo diario además de las negociaciones permanentes con las autoridades de la política científica. Todo lo cual condujo, aun con retrasos, a la inauguración del centro en 1975. Las principales dificultades eran económicas, nunca parecía haber suficiente presupuesto para el gran laboratorio que Ochoa hubiera querido, diseñado siguiendo las sugerencias técnicas de la consultora de arquitectos e ingenieros Haines Lunberg \& Waehler de los Estados Unidos, que había asesorado previamente en la construcción del centro de investigación que la empresa farmacéutica Hoffman-La Roche había creado junto a sus oficinas centrales en Nutley, Nueva Jersey, en 1969. La minuta de esta empresa fue pagada por los Estados Unidos ${ }^{12}$.

Ochoa deseaba contar con un centro técnicamente dotado, que le permitiera permanecer en el centro de la producción de conocimiento, como lo estaba entonces en la Universidad de Nueva York y lo estaría después en el Instituto Roche. «Dados los años que yo llevaba en Estados Unidos (unos treinta) y las extraordinarias facilidades de que disfrutaba allí para mi labor académica e investigadora era muy dudoso que nada me persuadiese a realizar un cambio» ${ }^{13}$. Su apoyo fue esencial para lograr la ayuda de la National Science Foundation a un proyecto firmado 
por Eladio Viñuela, Eduardo Torroja y Carlos Asensio en $1971^{14}$, el mismo año en el que se aprobó la construcción del Laboratorio Europeo de Biología Molecular con la participación de España. Las ayudas de la National Science Foundation fueron adjudicadas a grupos investigadores concretos, muchos de los cuales no llegaron nunca a formar parte del CBM, y que conservaron para sí las ayudas en el centro en el que permanecieron, el Centro de Investigaciones Biológicas, el cual, con tal motivo, mejoró considerablemente sus dotaciones técnicas. Asi pues, los recortes afectaron al número de investigadores e investigadoras que habrían de formar parte del personal científico del nuevo centro.

La dificultad principal para llevar a término el proyecto del CBM fue política, primero, y finalmente, también económica, otra vez. Porque los avatares de la historia política acompañaron a la comunidad científica, sucesivamente apoyaron, detuvieron y recuperaron considerablemente mermado en sus ambiciones económicas, técnicas y humanas aquel gran proyecto diseñado con los auspicios de Ochoa.

Ochoa perdió parte de protagonismo en el proyecto del CBM en cuanto Villar Palasí fue cesado en 1972. La copiosa documentación presentada en el Ministerio de Educación poco antes del cese quedó allí detenida, como lo estuvo el proyecto. Una dificultad política que se superó cuando volvió a cambiar el ministro de Educación y Ciencia, tras el asesinato del presidente del gobierno, el almirante Carrero Blanco. El nuevo jefe del ejecutivo, Rafael Arias Navarro, nombró ministro de Educación a Cruz Martínez Esteruelas y éste, a su vez, a Federico Mayor Zaragoza, subsecretario. El proyecto se recuperó, ya se ha dicho que con unos medios económicos muy mermados, bajo la dirección de Mayor Zaragoza. Catedrático de Bioquímica de la Universidad Autónoma de Madrid, consiguió de esa Universidad la cesión de algunos locales de la Facultad de Ciencias. Aquellas condiciones apartaron a Ochoa del proyecto. Pero para la comunidad científica española dedicada a la bioquímica y a la biología molecular, el proyecto era demasiado importante como para dejarse detener por recortes presupuestarios de última hora. Las competencias en materia de política científica del ministerio de Martínez Esteruelas eran desempeñadas por Mayor Zaragoza. Entre Viñuela y Mayor se estableció apoyo mutuo. Compartían un objetivo y se repartían la responsabilidad: Mayor tenía la política y Viñuela, por su parte, la científico-técnica. Actuaron de mutuo acuerdo y eso hizo posible un CBM cuyas reducidas dimensiones decepcionaron a Ochoa, pero que ha sido uno de los centros más influyentes en el devenir de las investigaciones en bioquímica y en biología molecular en España. 
En ese tiempo, a partir de la ejecución del I Plan de Desarrollo, el Fondo Nacional para el Desarrollo de la Investigación Científica, y durante la del II Plan (1964-1974), se repartieron entre muchos departamentos universitarios las primeras ayudas destinadas a la dotación científico-técnica de grupos de investigación y se reconoció explícitamente la conveniencia de promover una universidad investigadora ${ }^{15}$. Franco aún vivía y España seguía siendo una dictadura, aunque la organización social, profesional y económica estaba sujeta a influencias adicionales a las que el Jefe del Estado ejercía en el Consejo de Ministros.

La influencia internacional fue esencial. Los Estados Unidos tuvieron un papel principal en ello. Las ayudas económicas que la actividad científica española recibió de los Estados Unidos debe considerar, en su mayor parte, fruto del Acuerdo firmado por España con ese país, por el cual se establecieron las bases militares de Estados Unidos en España, mientras se le denegaba a esta su entrada en la OTAN. El interés estratégico de España fue el origen de lo que se denominó cooperación entre España y Estados Unidos, pero sobre todo, fue un poderoso agente de legitimación internacional del régimen, que contribuyó a su duración con fuerza comparable a la del terror creado por la represión durante la guerra civil y la primera década de la dictadura.

Pero la comunidad científica recibió los beneficios disponibles. La Agencia Internacional de la Energía Atómica subvencionó investigaciones del grupo de Gabriella Morreale y Francisco Escobar sobre endocrinología experimental por marcaje isotópico. Y la National Science Foundation sufragó buena parte de las dotaciones técnicas del CBM y, ya se ha dicho, también del CIB. Al menos desde el periodo de Lora-Tamayo, algunos científicos dedicados a la investigación biomédica se reunían con el ministro, con el presidente del CSIC, con el subsecretario y buscaban apoyos a los proyectos. El CBM fue solo uno de ellos, pero hubo otros: el Instituto de Biología Fundamental en Barcelona, el Instituto de Farmacología Española y el Instituto de Investigaciones Citológicas de Valencia, entre ellos.

El Instituto de Biología Fundamental fue consecuencia del acuerdo entre Juan Oró, químico español establecido en la Universidad de Houston y especializado en química prebiótica, con el equipo de Villar Palasí, y con el biólogo molecular catalán Jaume Palau. Se creó en un decreto de 1970 y se estableció inicialmente en Barcelona, en unos locales prefabricados, para trasladarse finalmente a su local definitivo en el campus de la Universidad Autónoma de Barcelona ${ }^{16}$. 
Pero el apoyo público, gubernamental, no fue el único que hizo posible la creación de centros de investigación en la España del desarrollo económico. El Instituto de Farmacología Española en Madrid y el Instituto de Investigaciones Citológicas de Valencia fueron un producto combinado de ayuda privada y política científica, producto de negociaciones que, finalmente, promovieron las experimentaciones biomédicas y resultaron influyentes en sus entornos académicos y geográficos.

La creación del Instituto de Farmacología Española-Fundación Marqués de Urquijo en 1950 fue consecuencia de una norma del CSIC por la que las industrias debían aportar a este organismo un porcentaje de sus ventas. La Compañía Española de Penicilinas y Antibióticos, una de las primeras industrias españolas dedicadas a la obtención y a la comercialización de antibióticos, junto al grupo de empresas quimico-farmacéuticas del Banco Urquijo, soslayó esa obligatoriedad creando un instituto que debía proporcionar apoyo técnico a ese grupo de empresas mientras simultáneamente hacía posible la investigación en neurofisiología. El IFE es inseparable de la personalidad de su director, el catedrático de Fisiología de la Universidad de Madrid Antonio Gallego, que combinó sus intereses científicos con el servicio técnico a las empresas del Urquijo y dio a la Universidad de Madrid una escuela de neurofisiología que ha tenido amplia influencia en el desarrollo de las investigaciones y de la docencia de esa disciplina en España. Desde 1954 sus locales estuvieron en la Facultad de Medicina de la Universidad de Madrid. A partir de 1953 editó su propia revistas, Anales del Instituto de Farmacología Española. El IFE marcó pautas en materia de política científica, si bien en una tendencia que tardaría en considerarse como parte sustancial de ésta, y era precisamente el apoyo privado a la investigación. Fue fruto de una compleja negociación, permanente, con las autoridades académicas de la Universidad de Madrid y del Consejo Superior de Investigaciones Científicas, los más influyentes y distinguidos de entre sus miembros formaban parte del patronato o del comité asesor del IFE. Este logró en los años 60 una de las muy escasas ayudas que la Fundación Rockefeller concedió a España durante el franquismo para los trabajos en neurofisiología de Gallego, que permitieron mejorar las dotaciones técnicas de este centro ${ }^{17}$.

El Instituto de Investigaciones Citológicas se había creado en Valencia a partir de un Laboratorio de Citología que dirigía el citólogo Gerónimo Forteza. Su prestigio científico hizo posible una importante ayuda de la Caja de Ahorros y Monte de Piedad de Valencia, sumada a otras previas de menor cuantía aportadas por la Fundación Marqués 
de Urquijo, del ministerio de Educación, del CSIC, que lo convirtieron en el Instituto de Investigaciones Citológicas en 1966. Forteza contó desde 1966 y hasta 1973 con apoyo de la Junta de Energía Nuclear para investigar sobre precursores radiactivos aplicados a la biología celular. En 1972 se inauguraron los nuevos locales del Instituto, donde se encuentra actualmente, en la calle Amadeo de Saboya, en Valencia ${ }^{18}$. La Caja de Ahorros de Valencia es el principal agente financiador de este centro, que está considerado en Valencia como pionero en experimentación biomédica en la región y ha tenido amplia influencia en la promoción de esta área de investigación en la Comunidad Valenciana. La política de recuperación de científicos establecidos en el extranjero emprendida por el equipo de Villar Palasí contribuyó, por su parte, a que Santiago Grisolía, uno de los primeros estudiantes postdoctorales de Ochoa en la Universidad de Nueva York, volviera a España para dirigirlo.

$\mathrm{Si}$, tras un lagro y complejo proceso de negocación, que incluyó a tres ministros de Educación —José Luis Villar Palasí, Julio Rodríguez Martínez y Cruz Martínez Esteruelas- se inauguró el Centro de Biología Molecular Severo Ochoa en la Facultad de Ciencias de la Universidad Autónoma de Madrid en septiembre de 1975, fue a consecuencia de la permanente negociación establecida entre las autoridades políticas y la comunidad científica dedicada a investigaciones biomédicas; en este caso, fue fundamental el papel de Eladio Viñuela ${ }^{19}$. Para cuando estaba previsto, no se sabía con certeza si Ochoa aceptaría inaugurar las instalaciones preparadas para tal acontecimiento.

La crisis económica provocada por el alza de los precios del petróleo desde los primeros años 70 afectó inmediatamente a la frágilmente dotada comunidad de investigación española. Las personas que formaban parte de ella, expertas en distintas áreas, alzaron entonces la voz, y tuvieron su eco, en la prensa española y en las revistas científicas extranjeras de máxima difusión, como fue el caso de Nature. Como la misma Nature había seguido en 1969 los sucesos políticos que llevaron a decretar el estado de excepción en toda España en el primer trimestre de 1969 y que pusieron en peligro la celebración de un congreso internacional de Bioquímica previsto en Madrid para el mes de abril de ese año. La comunidad científica española ha tenido en el extranjero apoyos importantes para llevar a cabo su trabajo, aunque en aquella ocasión se viviera como amenaza las expresiones políticas contra la dictadura manifestadas por algunas sociedades bioquímicas europeas.

Así pues, en tiempos de apoyos político-científico comparativamente mayores, la comunidad científica a través de algunos de sus miembros 
actuaba asesorando en la toma de decisiones. $\mathrm{Y}$ en los periodos de crisis reclamaba el apoyo que tuvo y recordaba, como ha hecho recientemente, que hubo tiempos en los que los medios eran mayores, o la distribución de apoyo económico se realizaba con menos retrasos. Pero todas aquellas personas de entre las mujeres y los hombres que componían la comunidad científica española que lograron mantener su trabajo en plena crisis de los 70 , y en otras posteriores, han emergido de ellas convertidas en autoridades científicas reforzadas por su capacidad investigadora y legitimadas para sucesivas reclamaciones de mayor apoyo político, presupuestario y social para la investigación en España.

Las políticas públicas que hicieron posibles los centros mencionados, de muy distinto carácter entre sí - las políticas y los centros- refieren permanentemente al papel jugado, en su creación, de los científicos que lideraron cada uno de los proyectos. Su trabajo científico era la principal legitimación para sus ambiciones en el logro del apoyo público a la investigación en las áreas biomédicas en España. Su prestigio estaba basado en lo que aprendieron en el extranjero y en lo que obtuvieron de agencias extranjeras de financiación. Los National Institutes of Health, el Jane Coffin Fund y la Fundación Rockefeller contribuyeron a esa legitimación a través de la concesión de las correspondientes ayudas.

Ochoa tuvo su protagonismo: un Nobel de medicina nacido en España se convirtió en bandera principal en las negociaciones con las autoridades políticas para la obtención de creciente apoyo público a la bioquímica y a la biología molecular. Y si la neurofisiología española no tuvo su Nobel, la sombra alargada de la escuela de Cajal terminó por desempeñar su papel y por muy diversas vías y con apoyos adicionales -entre ellos la creciente dedicación a las neurociencias de la comunidad científica extranjera - se convirtió en otra de las áreas en las que España hace contribuciones intensas y extensas desde diversos puntos de la geografía española.

La influencia internacional se dejó sentir en las políticas: la OCDE emitió sus informes sobre la investigación científica y técnica en España (OCDE, 1966 y 1974) y sus recomendaciones serían recogidas, al menos en parte, porque había un grupo de especialistas preparados para contribuir a su establecimiento, en los casos mencionados aquí con negociaciones directas con la autoridad política. Además de Ochoa, Juan Oró, Jaume Palau, Margarita Salas, David Vázquez, Ángel Martín Municio, Alberto Sols, Carlos Asensio, y Eladio Viñuela, por citar a algunos de los más reconocidos, contribuyeron a ello y al actuar de 
mediadores entre la comunidad experta y la autoridad política, sirvieron de asesores a los sucesivos apoyos públicos, en forma de subvenciones y becas de formación de personal investigador, que los gobiernos españoles manifestaron e hicieron efectivos a partir de mediados de los años 1960. Entre todos modelaron la acción gubernamental española en materia de política científica y deben considerarse el origen del hecho de que el Plan Nacional de I+D+I aprobado en 1999 haya hecho de las biociencias una de las áreas que más apoyo económico debería estar recibiendo y recibiría en los próximos dos años si se cumplen las previsiones allí recogidas.

\section{Agradecimientos}

Estoy en deuda con Jean-Jacques Salomon, que me ha proporcionado valiosas informaciones sobre el origen de la política científica en Europa tras la Segunda Guerra Mundial, y con Margarita Salas, Ricardo DíezHochleitner y Juan Oró, por haber compartido sus recuerdos sobre las negociaciones destinadas a hacer posible la vuelta de Severo Ochoa a España. Agradezco a Jesús Ávila y a Emilio Muñoz sus comentarios a una versión previa de este artículo.

\section{Notas}

1 Uno de los primeros y más influyentes trabajos sobre política científica es Ina Spiegel-Rosling y Derek de Solla Price (eds.)(1977): Science, technology and society: a cross disciplinary perspective (London: Sage). Para una revisión, véase Philip Gummett (1992): «Science and technology Policy", en Enciclopedia of Government and Politics, Mary Haekesworth y Maurice Kogan (eds.), vol. 2, págs. 759-776. Una historia de la política científica en España está en Luis Sanz Menéndez (1997): Estado, ciencia y tecnología en España 1939-1997 (Madrid: Alianza).

2 Ángel Viñas 1981. Los pactos secretos de Franco con los Estados Unidos. Bases, ayuda económica, recortes de soberanía (Barcelona: Grijalbo); Fernando Guirao (1998): Spain and the Reconstruction of Western Europe 1945-1957 (Londres: MacMillan); Antonio Moreno (1998): Franquismo y construcción europea (1951-1962) (Madrid: Tecnos).

3 Ya me he referido a ello de manera monográfica en otro trabajo, M.J. Santesmases (2000): "Severo Ochoa and the biomedical sciences in Spain under Franco", Isis 91 (4) (en prensa).

4 Bruno Latour (1997): La vida del laboratorio, (Madrid: Alianza) versión española de Eulalia Pérez Sedeño.

5 Philippe Laredo (1999): Report on the development of a reproducible method for the characterisation of a large set of research collectives (mimeo). 
6 Hay casos comparables en la historia reciente de la construcción de políticas científicas. Por ejemplo, Ronald Brickman y Arie Rip (1979): "Science Policy Advisors Councils in France, the Nederlands and teh United States, 1955-1977: A comparative analysis", Social Studies of Science, 9: 167-198. John Krige (1990): "Scientists as policymakers: British Physicists 'advice' to their Government on Membership of CERN", en Tore Frängsmyr (ed): Solomon's House revisited. The organisation and institutionalisation of science (Canton, Mass.: Science History Publications), 270-291.

7 Ricardo Díez-Hochleitner (1992): "La reforma educativa de la LGE de 1970. Datos para una crónica", Revista de Educación: La Ley General de Educación veinte años después, número extraordinario, pp. 261-278.

8 Sobre los las investigaciones bioquímicas en España, véase M.J. Santesmases y E. Muñoz (1997): Establecimiento de la bioquímica y de la biología molecular en España (Madrid: Fundación Ramón Areces) y M.J. Santesmases (1998): Alberto Sols (Alicante: Instituto de Cultura Juan Gil-Albert).

9 Sobre Carlos Asensio, véase Asensio, C. (1987) Cartas desde América (Oviedo: Caja de Ahorros de Asturias).

10 Los detalles sobre las ayudas de Estados Unidos a biólogos moleculares españoles están en M.J. Santesmases (1997): «Tradición y modernización: Aspectos cognitivos y socisles en los inicios de lá biología molecular en España", Arbor 614, 79-110.

11 Sobre Mayor Zaragoza, véase J. Bardavío (1994): Federico Mayor Zaragoza (Barcelona: Círculo de Lectores).

12 Severo Ochoa (1988): «El Centro de Biología Molecular: Los primeros años», $A b c, 5$ de marzo de 1988.

13 Ibid.

14 C. Asensio, E. Torroja, E. Viñuela, May, 1971, "A preliminary proposal to the National Science Foundation from the Institute and Graduate School of Molecular Biology (A joint project of the Autonomous University of Madrid and the Higher Council of Scientific Investigations). Archivo Alberto Sols.

15 Presidencia del Gobierno, CAICYT (1975): El Fondo Nacional para el desarrollo de la Investigación Científica (Madrid: Presidencia del Gobierno).

16 Sobre el IBF, véase M.J. Santesmases (1997): "Tradición y modernización", op. cit.

17 M.J. Santesmases (1999): Antibióticos en la autarquía: banca privada, industria faramacéutica, investigación científica y cultura liberal en España, 1940-1960 , documento de trabajo (Madrid: Fundación Empresa Pública).

18 “Instituto de Investigaciones Citológicas, Centro Superior de Investigación, Obra Social de la Caja de Ahorros y Monte de Piedad de Valencia•, editado por el Instotuto de Investigaciones Citológicas, Valencia, 1974.

19 Jesús Ávila, Carlos López-Otín y Manuel Perucho (eds.) (1998): Fago 29 y los orígenes de la Biología Molecular en España (Madrid). 


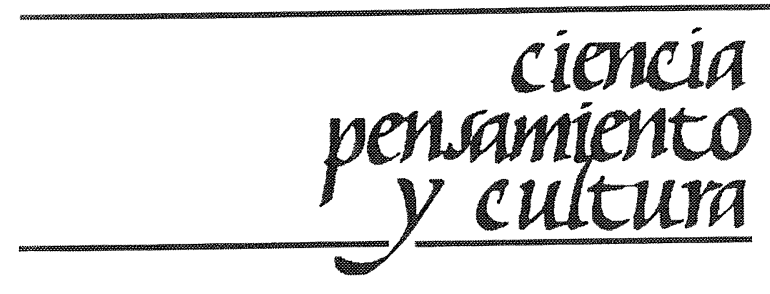

De próxima publicación:

LA MUJER EN EL ARTE

Carmen Rocamora (Editora)

La Revista Arbor está incluída en el apartado de Arte y Humanidades del CITATION INDEX

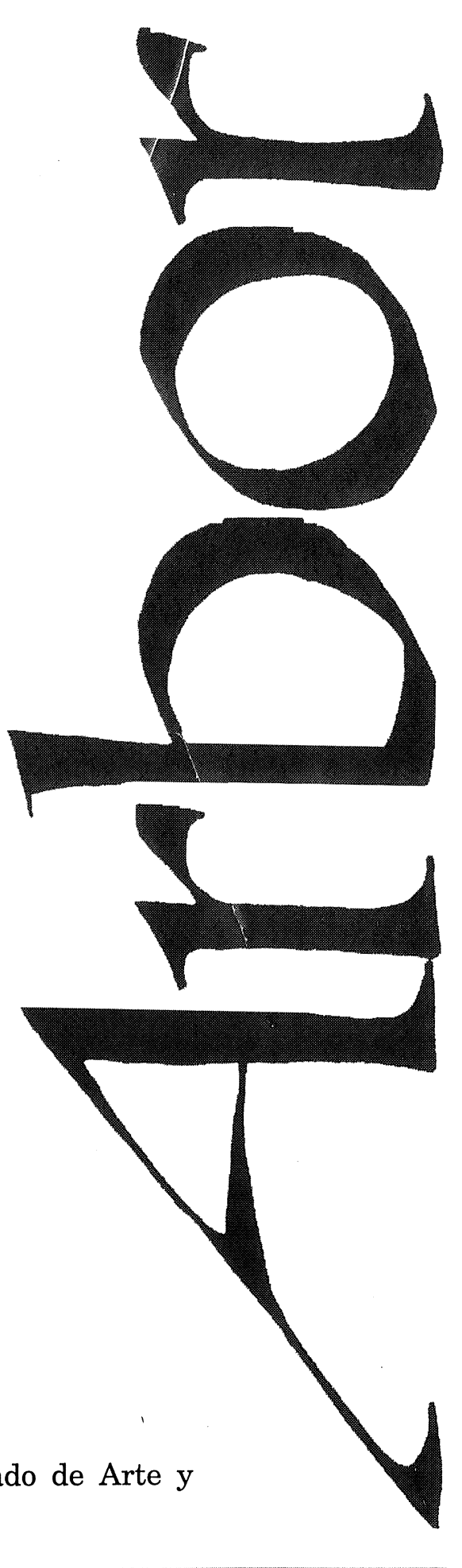

